\title{
Spin Physics: Session Summary
}

\author{
Daniël Boer ${ }^{1}$, Delia Hasch ${ }^{2}$ and Gerhard Mallot ${ }^{3}$ \\ 1- Vrije Universiteit Amsterdam - Department of Physics and Astronomy \\ De Boelelaan 1081, NL-1081 HV Amsterdam - The Netherlands \\ 2- INFN Laboratory Nazionali di Frascati \\ Via Enrico Fermi 40, I-00044 Frascati - Italy \\ 3- CERN, CH-1211 Geneva 23 - Switzerland
}

We summarize the main results of the spin physics Working Group Session of DIS 2007,
the 15th International Workshop on "Deep-Inelastic Scattering and Related Subjects".

\section{Introduction}

Many spin physics experiments have been performed in recent years and many new exciting results have been reported at DIS 2007 [1], which will be highlighted in this summary. Also on the theory side many new results were reported, especially regarding transverse spin effects which are most challenging. Recent years have seen quite some unexpected developments concerning so-called TMDs, transverse momentum dependent parton distributions, and we can look forward to more such developments over the coming years. Therefore, this summary is very much a snapshot of the current status.

This summary is split into three main parts. We start with longitudinal spin physics, most notably, experimental results on gluon polarization. We proceed with transverse spin, which is mainly focused on transverse spin asymmetries and the possible explanation in terms of TMDs. The third and last part is about exclusive processes and generalized parton distributions, which provide more detailed information about the spatial distribution of partons inside hadrons. This spatial distribution is often probed using spin asymmetries and recent developments have started to point to a connection between GPDs and TMDs. A very interesting development.

\section{Longitudinal spin}

The discovery by the European Muon Collaboration [2] that the first moment $\Gamma_{1}^{p}$ of the spin-dependent structure function $g_{1}^{p}$ of the proton

$$
\Gamma_{1}^{p / n}\left(Q^{2}\right)=\int_{0}^{1} \mathrm{~d} x g_{1}^{p / n}\left(x, Q^{2}\right)=\frac{1}{36}\left(4 \Delta \Sigma \pm 3 \Delta q_{3}+\Delta q_{8}\right)\left(1+\frac{\alpha_{s}}{\pi}\right)+\mathcal{O}\left(\alpha_{s}^{2}\right)
$$

is much smaller than expected implies that the total contribution of the quark spins to the nucleon spin $\Delta \Sigma \equiv \Delta u+\Delta d+\Delta s$ is small. Here $\Delta q=\left(q_{+}-q_{-}\right)+\left(\bar{q}_{+}-\bar{q}_{-}\right)$is the difference of the number of quarks and antiquarks of flavor $q$ with positive and negative helicity and $\Delta q_{3} \equiv \Delta u-\Delta d$ and $\Delta q_{8} \equiv \Delta u+\Delta d-2 \Delta s$ are known from $\beta$ decays.

HERMES presented the final analysis of their $g_{1}^{p}$ and $g_{1}^{d}$ measurements [3] and COMPASS showed new, very precise deuteron data [4] (Fig. 1). Both collaborations evaluated $\Delta \Sigma$ from their deuteron data with $Q^{2}>1 \mathrm{GeV}^{2}$ yielding $\Delta \Sigma=0.330 \pm 0.025$ (exp.) \pm 0.028 (evol.) \pm 0.011 (theo.) at $Q^{2}=5 \mathrm{GeV}^{2}$ from HERMES and $\Delta \Sigma=0.35 \pm 0.03$ (stat.) \pm 0.05 (syst.) at $Q^{2}=3 \mathrm{GeV}^{2}$ from COMPASS. The results are in excellent agreement. The value for 


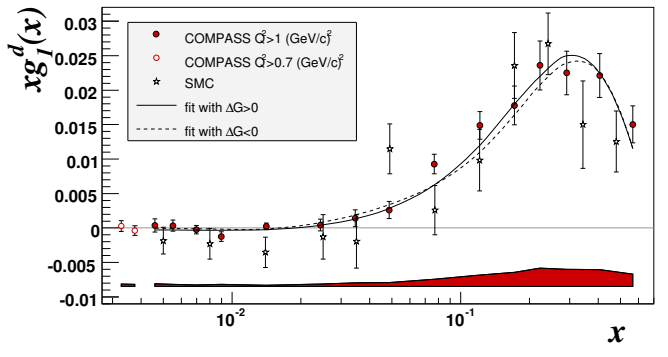

Figure 1: The deuteron structure function $x g_{1}^{d}$ as function of $x$ from COMPASS [4]. Also shown are QCD fits with positive and negative $\Delta G$.

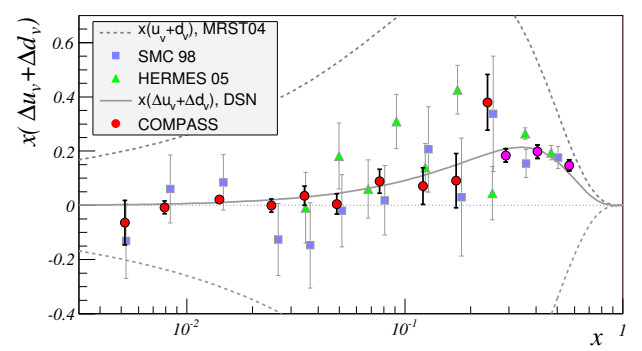

Figure 3: The valence quark distribution $x\left(\Delta u_{v}+\Delta d_{v}\right)$ as function of $x$ from SIDIS obtained in LO and evolved to $Q^{2}=$ $10 \mathrm{GeV}^{2}$ [7] using the PDFs of Ref. [8].
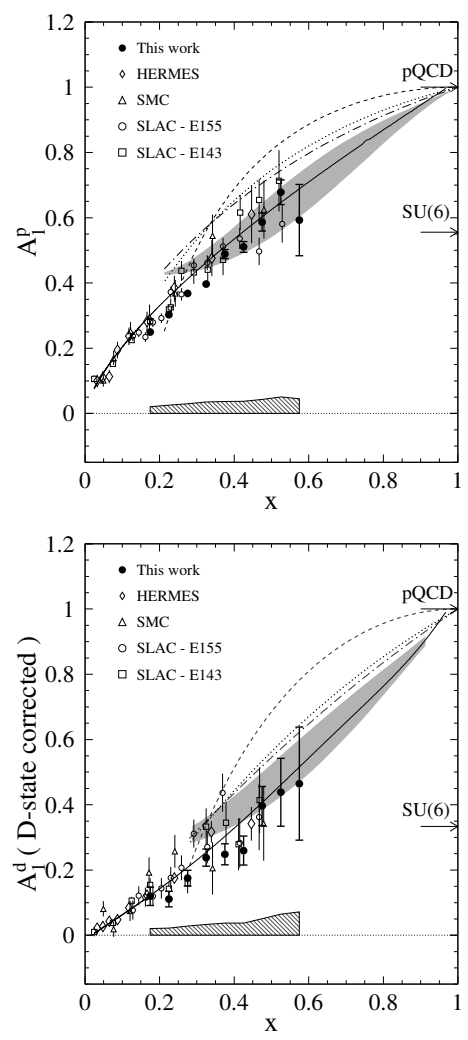

Figure 2: Asymmetry $A_{1}$ for the proton (top) and the deuteron (bottom) from CLAS EG1 for $Q^{2}>1 \mathrm{GeV}^{2}$ and $W>$ $2 \mathrm{GeV}$ [5].

$\Delta \Sigma$ is somewhat larger than the original EMC result of $\Delta \Sigma=0.12 \pm 0.17$, which was given at a larger scale $Q^{2}=10.7 \mathrm{GeV}^{2}$. All results are consistent with each other upon taking evolution into account. Therefore, the conclusion that the quark spins contribute little to the nucleon spin remains valid.

CLAS from JLAB showed a wealth of proton and deuteron $g_{1}$ data covering the range $0.05<Q^{2}<5 \mathrm{GeV}^{2}$. For $Q^{2}>1 \mathrm{GeV}^{2}$ the range $0.15<x<0.58$ is covered [5] (Fig. 2). The spin structure in the resonance region and the Burkhardt-Cottingham sum rule were explored by the Hall-C experiment E01-006 [6].

Semi-inclusive DIS (SIDIS), in which in addition to the scattered lepton a hadron is observed, can be analyzed in terms of the valence quark helicity distributions $\Delta q_{v}$. New COMPASS deuteron data obtained in leading order (LO) and using a fragmentation-function independent method [7] are shown in Fig. 3 together with previous data. They disfavour a flavor-symmetric quark sea with $\Delta \bar{u}=\Delta \bar{d}=\Delta s=\Delta \bar{s}$. 

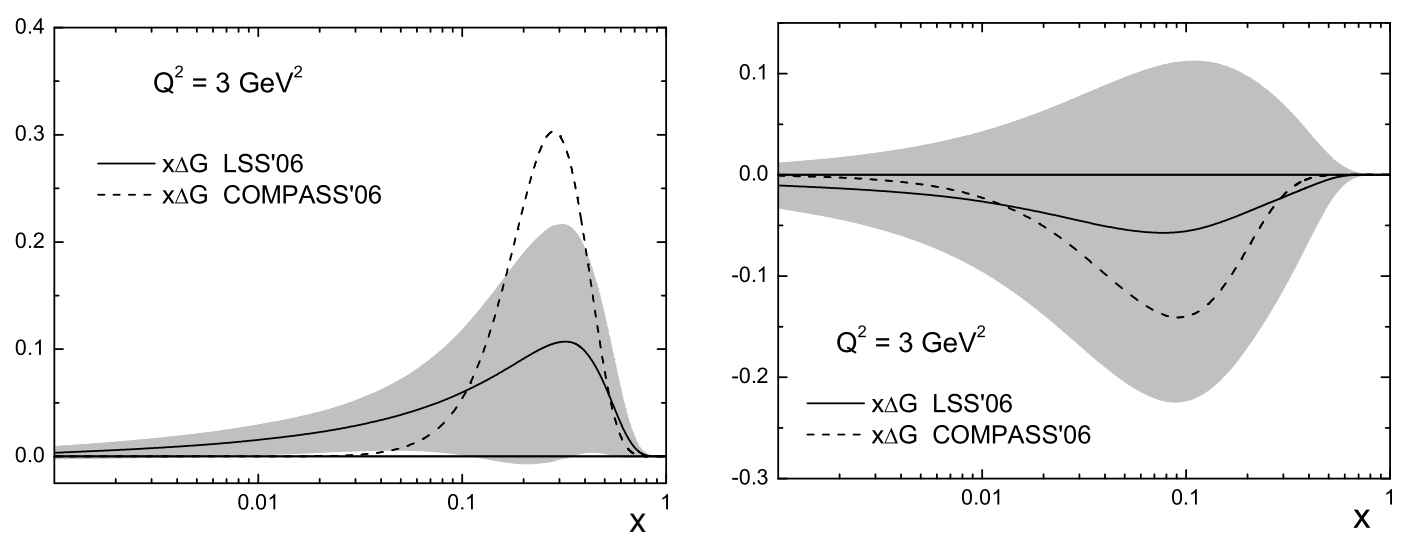

Figure 4: Solutions for positive (left) and negative (right) gluon polarizations $x \Delta G(x)$ as function of $x$ from LSS [10] (solid line) with uncertainties. The dashed line shows the corresponding COMPASS fits [4].

Apart from the contribution of the quark spins $\Delta \Sigma$, the nucleon spin sum rule

$$
\frac{1}{2}=\frac{1}{2} \Delta \Sigma+\Delta G+L_{z}
$$

receives contributions from gluon spins $\Delta G=\int \mathrm{d} x\left(G_{+}-G_{-}\right)$and from orbital angular momentum $L_{z}$, which must compensate for the smallness of $\Delta \Sigma$. Experiments start to obtain information on the gluon polarization $\Delta G$, although uncertainties are still large. The gluon polarization can be studied in polarized DIS and SIDIS and in $\vec{p} \vec{p}$ interactions.

Inclusive DIS is sensitive to $\Delta G(x)$ through the $Q^{2}$ evolution of $g_{1}$. However, the lack of a polarized lepton-proton collider limits the kinematic range of $g_{1}$ to the fixed-target domain at moderate $x$ and $Q^{2}$. The status of QCD fits to the world $g_{1}$ data from CERN, DESY, JLAB and SLAC were reviewed by J. Blümlein. He also summarized the status of $\alpha_{s}\left(M_{Z}^{2}\right)$ as obtained from DIS up to NNNLO for the unpolarized case and NLO for the polarized case [9]. Although the precision of $\alpha_{s}$ from polarized DIS can not yet reach that from the unpolarized data, the precision is remarkable.

As example for the present status of the QCD analyses we show the recent one by the LSS group [10], which takes into account the latest data from COMPASS [4] and CLAS [5]. They obtain three equally good solutions for positive, negative and sign-changing gluon polarization. The positive and negative solutions are compared in Fig. 4 with the solutions obtained by COMPASS in a similar analysis. At present even the sign of the gluon polarization cannot be determined from DIS data, however all fits yield a small value for the first moment $|\Delta G| \lesssim 0.3$ at $Q^{2}=3 \mathrm{GeV}^{2}$.

At small $Q^{2}$ standard DGLAP fits cannot be applied without considering higher-twist effects. The LSS group explicitly included such terms in their fits. The resulting higher-twist contributions are driven by the CLAS data [5]. Ermolaev focused on the small- $x$ aspects of the singlet part of $g_{1}$, in particular the resummation of the leading $\ln 1 / x$ terms [11]. He suggested to study the dependence on the invariant energy $2 P \cdot q$ rather than $Q^{2}$ to estimate the impact of the initial gluon density. 
More direct information on the gluon polarization can be obtained in SIDIS. Photongluon fusion (PGF) $\gamma g \rightarrow q \bar{q}$ leading to a quark-antiquark pair gives rise to a double-spin cross-section asymmetry proportional to the gluon polarization

$$
A_{\|}=R_{\mathrm{pgf}} a_{L L}^{\mathrm{pgf}} \frac{\Delta G}{G}+A_{\mathrm{bgd}}
$$

where $R_{\text {pgf }}$ is the fraction of PGF events and $a_{L L}^{\mathrm{pgf}}$ is the analyzing power of the PGF subprocess. For a particular measurement both, $R_{\text {pgf }}$ and the average $a_{L L}^{\text {pgf }}$, have to be estimated using Monte Carlo (MC) simulations. This introduces a model dependence in the determination of $\Delta G / G$. In the light-quark case the QCD-Compton process $\gamma q \rightarrow q g$ and the direct process $\gamma q \rightarrow q$ limit $R_{\mathrm{pgf}}$ to about $30 \%$, while for charmed quark pairs $R_{\mathrm{pgf}}$ is essentially unity. Here the challenges are the low production cross-section and the detection of open charm ( $D$ mesons). The most promising decay channel $D \rightarrow K \pi$ has a branching ratio of only $3.8 \%$ which implies that only one of the two charmed hadrons can be observed with reasonable statistics. Until now all analyses were performed in leading order.

HERMES determined $\Delta G / G$ from single high- $p_{T}$ hadron production asymmetries in four bins of transverse hadron momentum $p_{T}$ in the range $1.05 \mathrm{GeV}<p_{T}<2.5 \mathrm{GeV}$ using two methods [12]. Method I directly used the above equation for $A_{\|}$with $R_{\text {pgf }}\left(p_{T}\right)$ and $a_{L L}^{\mathrm{pgf}}\left(p_{T}\right)$ determined using a PYTHIA Monte Carlo simulation. In Method II two different parameterizations of $\Delta G / G$ were fitted to the measured asymmetries in the four $p_{T}$ bins. The gluon polarization is small and probed around $x \simeq 0.22$ at $\mu^{2}=1.35 \mathrm{GeV}^{2}$. The resulting value $\Delta G / G=0.071 \pm 0.034$ (stat.) \pm 0.010 (syst.) ${ }_{-0.105}^{+0.127}$ (model) is shown together with the fitted parameterizations and other data in Fig. 5.

COMPASS determined $\Delta G / G$ from the cross-section asymmetries for $D$ meson production in [13]. This method relies much less on Monte Carlo simulations but is limited in statistical precision. A neural network was used to estimate $a_{L L}^{\mathrm{pgf}}$ from the event kinematics on an event-by-event basis. The result $\Delta G / G=-0.57 \pm 0.41 \pm$ 0.17 (syst.) is compatible with zero and probes the gluon distribution around $\mu^{2}=$ $13 \mathrm{GeV}^{2}$ and $x=0.15$. This is also compatible with the most precise COMPASS result from light-quark pairs at $Q^{2}<1 \mathrm{GeV}^{2}$ of $\Delta G / G=0.016 \pm 0.058 \pm 0.055$ (syst.). All results from PGF in DIS are summarized in Fig. 5 and in Ref. [14].

$\Delta G$ can in principle also be obtained from polarized photoproduction of hadron pairs with high transverse momenta $\left(p_{T, 3}, p_{T, 4}\right)$. Hendlmeier presented NLO cal-

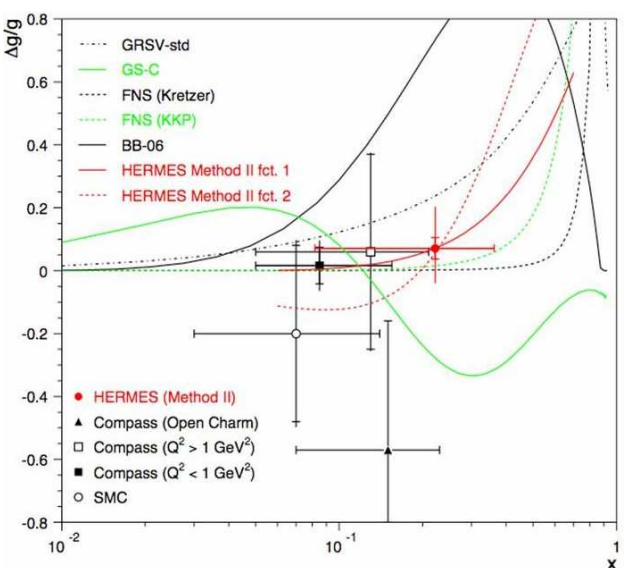

Figure 5: The gluon polarization $\Delta G / G$ as function of $x$. The new HERMES point lies at $\Delta G / G \simeq 0.07$ and $x \simeq 0.2$, where the two fitted parameterizations intersect. culations for this process with HERMES and COMPASS kinematics. The scale dependence for the cross-sections and asymmetries at NLO is generally not smaller than at LO. An interesting option is the reduction of the 

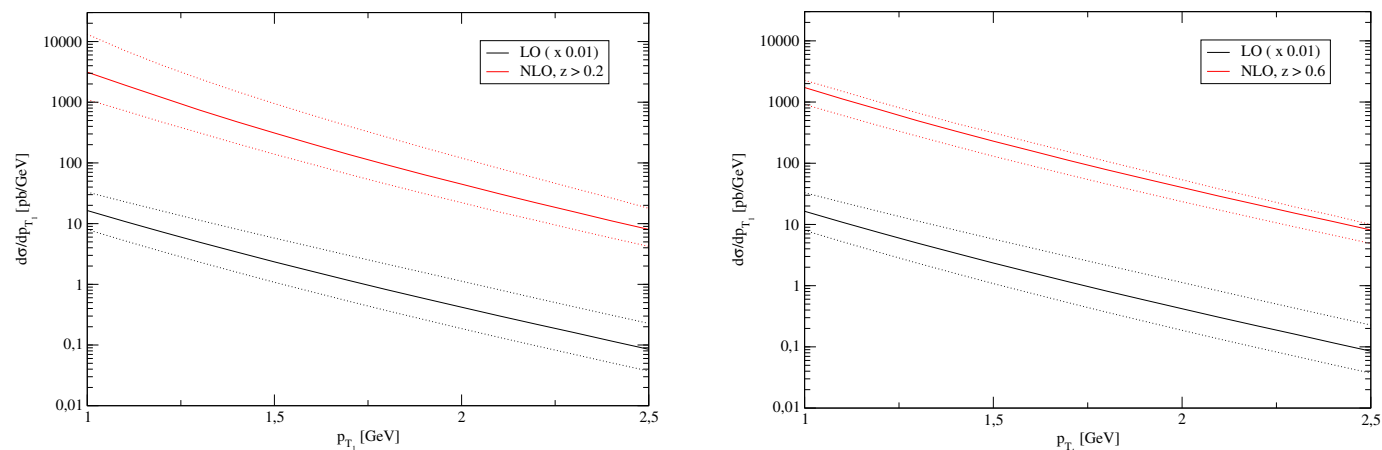

Figure 6: Scale dependence for the pair cross-section as function of the $p_{T}$ of the first hadron in LO (bottom curves) and NLO (top curves) for $z>0.2$ (left) and $z>0.6$ (right) and COMPASS kinematics. The scale $\mu$ is varied by a factor two around $\mu=p_{T, 3}+p_{T, 4}$, see the text for the definition of $z$.

scale dependence by cutting on a variable defined as $z=-\vec{p}_{T, 3} \cdot \vec{p}_{T, 4} / \vec{p}_{T, 3}^{2}$. This only works for the COMPASS kinematics (Fig. 6), while in the HERMES case the cut has little effect.

At RHIC cross-section asymmetries for longitudinally polarized $\vec{p} \vec{p}$ scattering at $\sqrt{s}=$ $200 \mathrm{GeV}$ were analyzed for several channels. PHENIX presented results for $\pi^{0}$ production [15]. The cross-section is well understood over seven orders of magnitude in NLO [16], as can be seen in Fig. 8. Their data favour a small gluon polarization and are compatible with the $\Delta G=0$ and the standard scenario of GRSV [17] in NLO (Fig. 7). Also a negative gluon polarization cannot be excluded. Future measurements at $\sqrt{s}=500 \mathrm{GeV}$ will remove the present ambiguity because of the decreasing relative importance of the quadratic term in $\Delta G$ with increasing $p_{T}$. Data taken in 2006 at $\sqrt{s}=62.4 \mathrm{GeV}$ (see Fig. 8 for the cross section measured by BRAHMS at this energy) will allow to probe higher $x_{\text {gluon }}$.

STAR presented longitudinal spin asymmetries for inclusive jet production [18] (Fig. 9) and pions [19] from the 2005 run. Again the cross-sections are well understood in NLO [20] and the data point to a rather small gluon polarization and negative values cannot be excluded. The precise data taken in 2006 will drastically improve the statistical precision.

All data suggest gluon polarization $|\Delta G| \lesssim 0.3$, where one has to keep in mind that the relevant scales for the various measurements vary. Although this value is by far smaller than the values around 2-3 predicted by some models assuming a restoration of $\Delta \Sigma$ to the Ellis-Jaffe value of 0.6 via the axial anomaly, it does not exclude that the gluon and quark spins make up the entire nucleon spin of $1 / 2$. Therefore, the importance of orbital angular momentum remains to be seen (further discussion on this topic can be found in section 4).

\section{Transverse spin}

In analogy to the axial charge $\Delta q$ the tensor charge $\delta q$ is defined as

$$
\left\langle P, S\left|\bar{\psi}_{q}\left[\gamma^{\mu}, \gamma^{\nu}\right] \gamma_{5} \psi_{q}(0)\right| P, S\right\rangle \sim \delta q\left[P^{\mu} S^{\nu}-P^{\nu} S^{\mu}\right]
$$

which arises for a transversely polarized proton. This fundamental charge $\delta q$ is the first Mellin moment of the so-called transversity distribution $h_{1}(x)$. It encodes completely new 

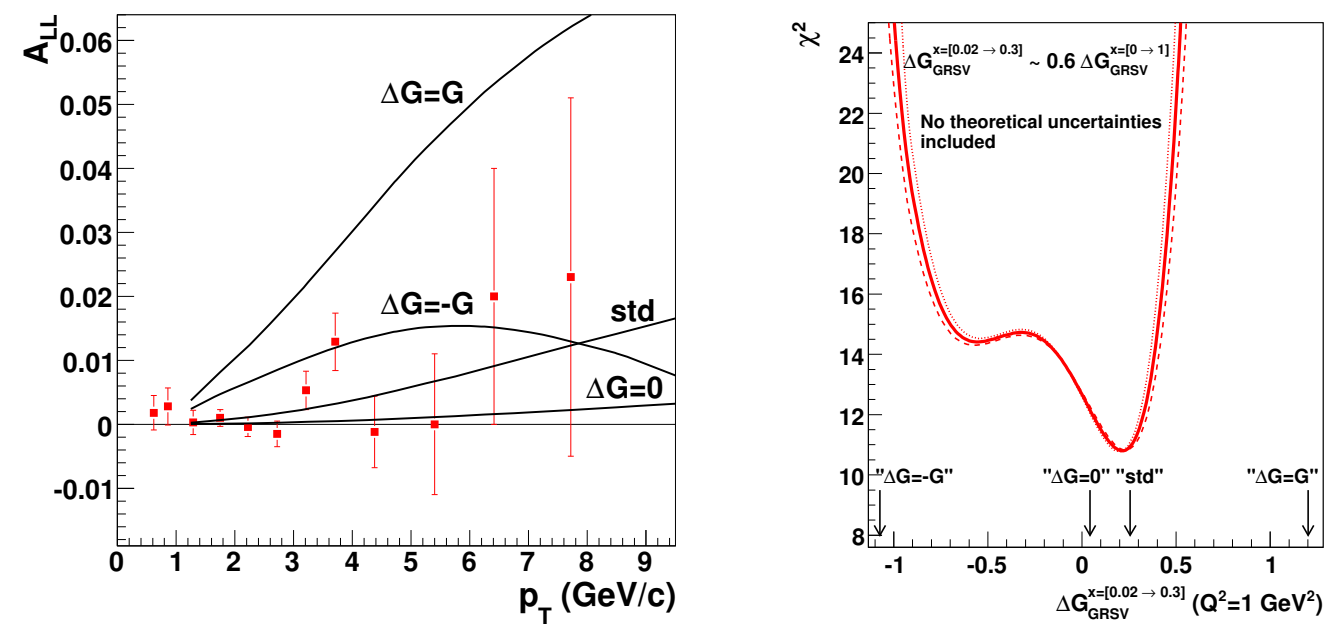

Figure 7: PHENIX $\pi^{0}$ asymmetry data [15]. Left: $A_{L L}$ as function of $p_{T}$. A scale uncertainty of $9.4 \%$ is not included. The curves correspond to the NLO predictions for various GRSV parameterizations [17]. Right: $\chi^{2}$ as function of $\Delta G_{\mathrm{GRSV}}$, only statistical errors are taken into account.
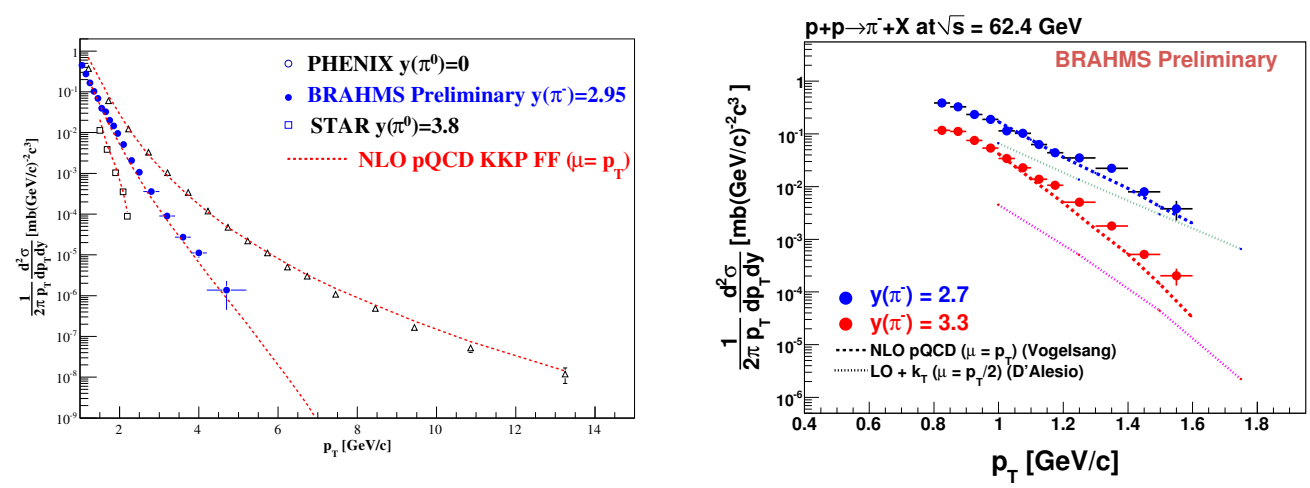

Figure 8: Cross-sections measured at RHIC. Left: Data by the PHENIX, STAR and BRAHMS experiments at $\sqrt{s}=200 \mathrm{GeV}$, compared with NLO pQCD predictions. Right: Similarly for data by the BRAHMS experiment at $\sqrt{s}=62.4 \mathrm{GeV}$.

information on the proton spin structure and is difficult, but not impossible to measure. Theoretically the most safe extractions can come from processes for which collinear factorization can be applied. In this case these are the following single and double transverse-spin asymmetries:

- $A_{T T}$ in $p^{\uparrow} \bar{p}^{\uparrow} \rightarrow \ell \bar{\ell} X$

- $A_{T}$ in various processes exploiting two-hadron fragmentation functions 

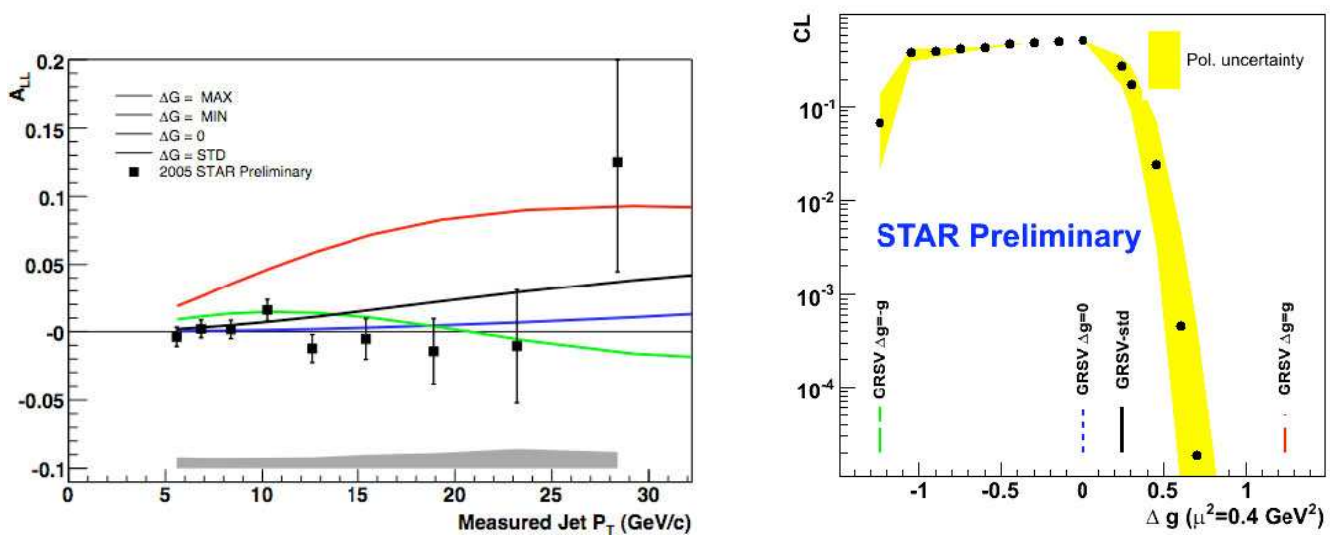

Figure 9: STAR inclusive jet asymmetry data. Left: $A_{L L}$ as function of the measured jet $p_{T}$. The scale uncertainty of $25 \%$ is not included in the shaded systematic error band. The curves correspond to the NLO predictions for various GRSV parameterizations [17]. Right: Confidence level as function of $\Delta G_{\mathrm{GRSV}}$.

The HERMES experiment has obtained the first non-zero transversity signal from the measurement of $A_{T}$ in the process $e p^{\uparrow} \rightarrow e^{\prime}\left(\pi^{+} \pi^{-}\right) X$ [21]. At DIS 2007 the COMPASS results on $A_{T}$ in $\mu d^{\uparrow} \rightarrow \mu^{\prime}\left(\pi^{+} \pi^{-}\right) X$ were presented: they are consistent with zero [22]. This is in line with the expectation that $h_{1}^{u} \approx-h_{1}^{d}$ leading to cancellations for a deuteron target. In the near future COMPASS will run with a proton target, allowing a check of the HERMES results. The two-hadron fragmentation functions themselves will be extracted in the future from BELLE data [23], which is crucial for the quantitative extraction of transversity from $e / \mu p^{\uparrow}$ or $p p^{\uparrow}$ processes. On the theory side, Radici discussed evolution equations for two-hadron fragmentation functions [24], which is an important issue when extracting transversity from a combination of two-hadron production observables measured in different experiments. Radici pointed out that the $R_{T}^{2}$ dependence (which is the square of the difference of the transverse momenta of the two hadrons) leads to a homogeneous evolution equation for the two-hadron fragmentation functions.

Hägler discussed the transverse spin structure of hadrons from lattice QCD with dynamical quarks, in particular more precise results on tensor GPDs (generalized parton distributions will be addressed further in section 4) [25], which may also shed light on transverse momentum dependent parton distributions, as will be discussed below.

Kawamura presented results [26] for $A_{T T}\left(Q_{T}\right)$ in the Drell-Yan process, which is proportional to $h_{1}$ times $h_{1}$. Soft gluon resummation was taken into account. Predictions for $p^{\uparrow} p^{\uparrow}$ at RHIC and J-PARC and for $p^{\uparrow} \bar{p}^{\uparrow}$ at GSI were given (Fig. 10). The latter observable displays a notably larger dependence on the scale $Q^{2}$ than the former.

Not all transverse spin asymmetries are associated with transversity though. Large single-spin asymmetries $\left(A_{N}\right)$ in $p p^{\uparrow} \rightarrow \pi X$ have been observed by several experiments (E704 Collab. ('91); AGS ('99); STAR ('02); BRAHMS ('05); ...). The observed asymmetries are left-right asymmetries, which means the pion distribution is left-right asymmetric depending on the transverse spin direction and the pion charge. 
$(\%)$

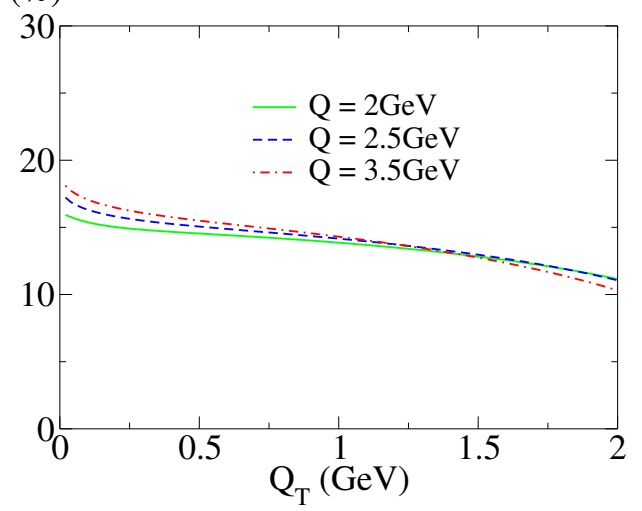

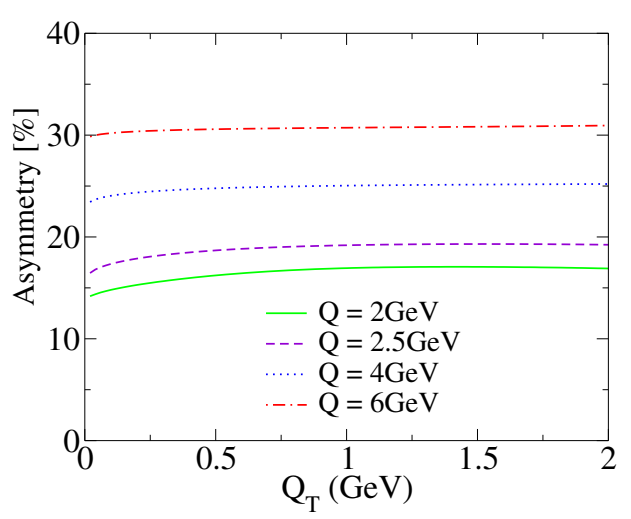

Figure 10: $A_{T T}\left(Q_{T}\right)$ predictions for $p^{\uparrow} p^{\uparrow}$ at J-PARC and $p^{\uparrow} \bar{p}^{\uparrow}$ at GSI $($ at $\sqrt{s}=10$ and 14.5 $\mathrm{GeV}$, resp.) [26].
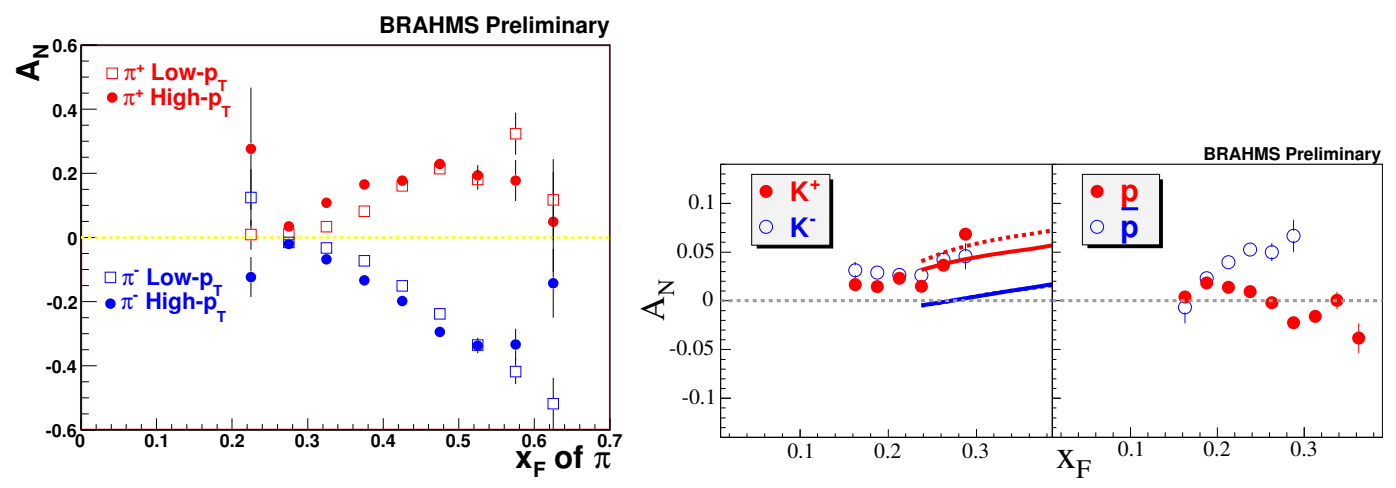

Figure 11: Single spin asymmetries measured by BRAHMS. Left: $A_{N}$ for $\pi^{ \pm}$as function of $x_{F}$, a high and low $p_{T}$ data comparison at $\sqrt{s}=62 \mathrm{GeV}$. Right: $A_{N}$ for $K^{ \pm}, p$ and $\bar{p}$ at $\sqrt{s}=200 \mathrm{GeV}$.

New $A_{N}$ measurements were presented at DIS2007. For example, Fig. 11 shows several single-spin asymmetries measured by BRAHMS [27]. PHENIX presented $A_{N}$ asymmetries for charged hadrons at mid rapidity as function of $p_{T}$ and for $J / \psi \rightarrow \mu^{+} \mu^{-}$at $x_{F} \approx \pm 0.1$; all are consistent with zero [28]. STAR presented $A_{N}$ asymmetries for forward $\pi^{0}$ 's and for larger $x_{F}(>0.4)$ also as a function of $p_{T}$, these are shown in Fig. 12 [29].

To understand the origin of these single-spin asymmetries a different explanation at the quark-gluon level is required than simply non-zero transversity.

One suggestion put forward is to describe $A_{N}$ at the twist-3 level, the so-called QiuSterman effect [30]. It involves a matrix element of the form

$$
G_{F} \sim\left\langle P, S_{T}\left|\bar{\psi}(0) \int d \eta^{-} F^{+\alpha}\left(\eta^{-}\right) \gamma^{+} \psi\left(\xi^{-}\right)\right| P, S_{T}\right\rangle
$$



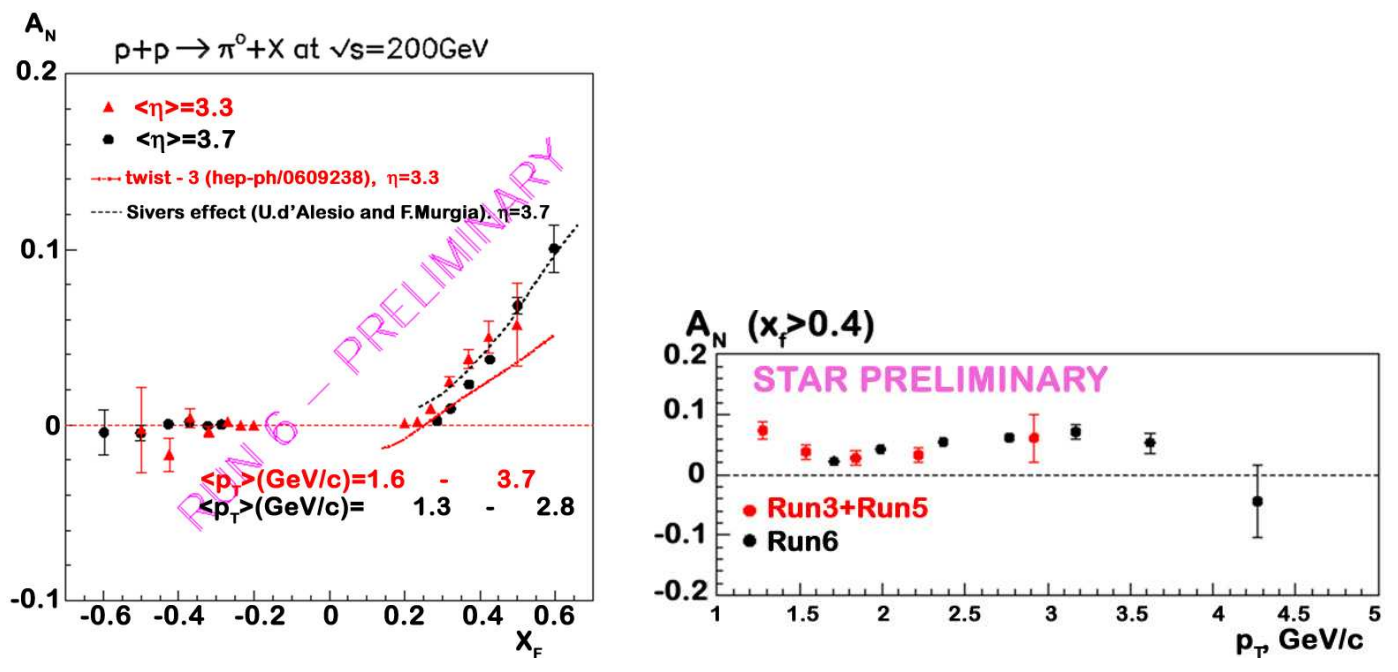

Figure 12: Single spin asymmetries measured by STAR. Left: $A_{N}$ for forward $\pi^{0}$ as function of $x_{F}$ at $\sqrt{s}=200 \mathrm{GeV}$. Right: $A_{N}$ for $x_{F}>0.4$ as a function of $p_{T}$.

This formalism applies at high transverse momentum of the pion. At DIS 2007 recent progress concerning this formalism was presented. Koike discussed the recent demonstration of twist-3 factorization and gauge invariance of the $A_{N}$ expression [31]. Tanaka presented a novel master formula for $A_{N}$ in various processes [32]. He showed that the twist-3 single-spin asymmetry can be obtained from the twist- 2 unpolarized cross-section. This provides a significant simplification of the calculation and an understanding of why always the combination $G_{F}-x d G_{F} / d x$ appears.

Another suggestion is to describe $A_{N}$ using transverse momentum dependent parton distributions (TMDs). TMDs arise from the natural extension of $x$ dependent functions to $x$ and $k_{T}$ dependent functions. But allowing for a dependence on $k_{T}$ also implies the appearance of new functions, such as the Sivers function [33] $f_{1 T}^{\perp}$ :

$$
f_{1}(x) \Longrightarrow f_{1}\left(x, \boldsymbol{k}_{T}^{2}\right)+\frac{\boldsymbol{P} \cdot\left(\boldsymbol{k}_{T} \times \boldsymbol{S}_{T}\right)}{M} f_{1 T}^{\perp}\left(x, \boldsymbol{k}_{T}^{2}\right) .
$$

Upon integration over transverse momentum the $k_{T}$-odd Sivers function $f_{1 T}^{\perp}$ drops out. Similarly, a chiral-odd TMD can arise that is also $k_{T}$-odd: $h_{1}^{\perp}$. In addition, the fragmentation function analogues $D_{1 T}^{\perp}$ and $H_{1}^{\perp}$ arise.

The Sivers effect can lead to a non-zero $A_{N}$ in $p p^{\uparrow} \rightarrow \pi X$, but also to azimuthal spin asymmetries in many different processes, such as in semi-inclusive DIS or back-to-back jets in $p p$ scattering. This allows to test the consistency of the many asymmetries described within this formalism.

In semi-inclusive DIS (Fig. 13) the Sivers function leads to a $\sin \left(\phi_{h}-\phi_{S}\right)$ asymmetry $\left(\propto f_{1 T}^{\perp} D_{1}\right)$, which can be distinguished from the Collins asymmetry $\sin \left(\phi_{h}+\phi_{S}\right)$ which arises with the transversity function $\left(\propto h_{1} H_{1}^{\perp}\right)$ [35]. Bacchetta presented the complete expressions of all 18 possible semi-inclusive DIS structure functions in terms of TMDs [34].

The first azimuthal spin asymmetry measurement was done by the HERMES Collaboration [36]. At DIS 2007 the latest HERMES and COMPASS results on the Sivers and Collins 


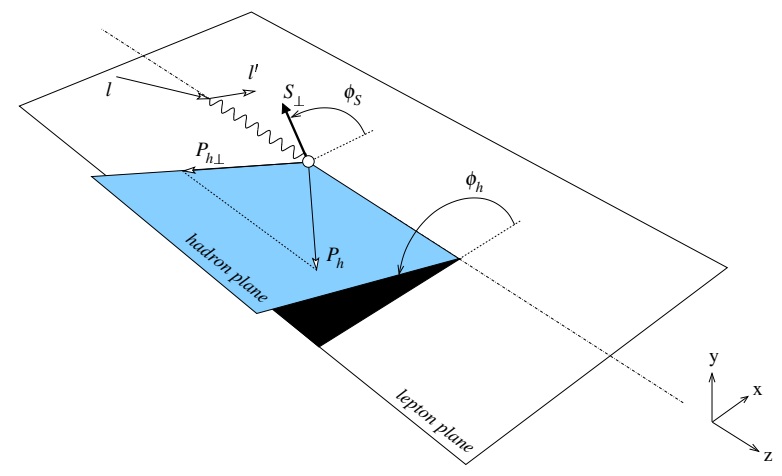

Figure 13: Kinematics of semi-inclusive DIS, with azimuthal angles $\phi_{S}$ and $\phi_{h}$ indicated [34].

asymmetries were presented. The HERMES data from 2002-2005 show large positive (negative) Collins asymmetries for $\pi^{+}\left(\pi^{-}\right)$[37], indicating that the Collins function $H_{1}^{\perp}$ for favored fragmentation is approximately equal in magnitude to unfavored fragmentation, but of opposite sign. For the Sivers asymmetry the $\pi^{+}$data show a significant non-zero asymmetry, but the $\pi^{-}$data are consistent with zero. The neutral pions follow the expectation from isospin symmetry for both types of asymmetry. The $K^{ \pm}$asymmetries have less statistical accuracy, but are similar to those for $\pi^{ \pm}$, although $K^{+}$shows even larger Sivers asymmetries than $\pi^{+}$. This may indicate that the sea contribution to the Sivers mechanism is of importance. COMPASS results on these and other asymmetries show that for the deuteron these asymmetries are all consistent with zero, indicating cancellations rather than small functions $[38,39]$.

As mentioned, the Sivers effect can also lead to a non-zero $A_{N}$ asymmetry for back-toback jet production in $p p^{\uparrow}$ scattering [40]. In general, the two jets are not exactly back-toback and an asymmetric distribution of one jet around the other may arise from the Sivers effect. This effect translates into a (generally smaller) left-right asymmetry for the bisector of the two jet directions. STAR results on the bisector left-right asymmetry are consistent with zero [42]. The data are also consistent with a recent prediction presented by Bomhof [41], based on Sivers function input from semi-inclusive DIS which probes mostly the large- $x$ part of the Sivers functions. One concludes that the smaller $x$ part that is probed in the back-toback jets Sivers asymmetry is likely to be small. However, another aspect that contributes to the suppression of the magnitude of the back-to-back jets Sivers asymmetry is that the color flow of the process makes it less sensitive to the Sivers function. It has been noted several years ago by Collins [43] that TMDs can exhibit a calculable process dependence, leading to the result that the Sivers function that enter the semi-inclusive asymmetry enters the analogous Drell-Yan asymmetry with opposite sign. This is due to the different color flows in the two processes. Bomhof and collaborators have found that the more hadrons are observed in a process, the more complicated the end result. At DIS 2007 Bomhof presented results [41] for $p^{\uparrow} p \rightarrow$ jet jet $\mathrm{X}$, included in Fig. 14 .

Also the Collins function can lead to asymmetries in other processes besides semiinclusive DIS. It leads to $\cos 2 \phi$ asymmetries in several processes, most notably in $e^{+} e^{-} \rightarrow$ $\pi^{+} \pi^{-} X$, which can be used to extract the Collins function [44]. This has been done using 

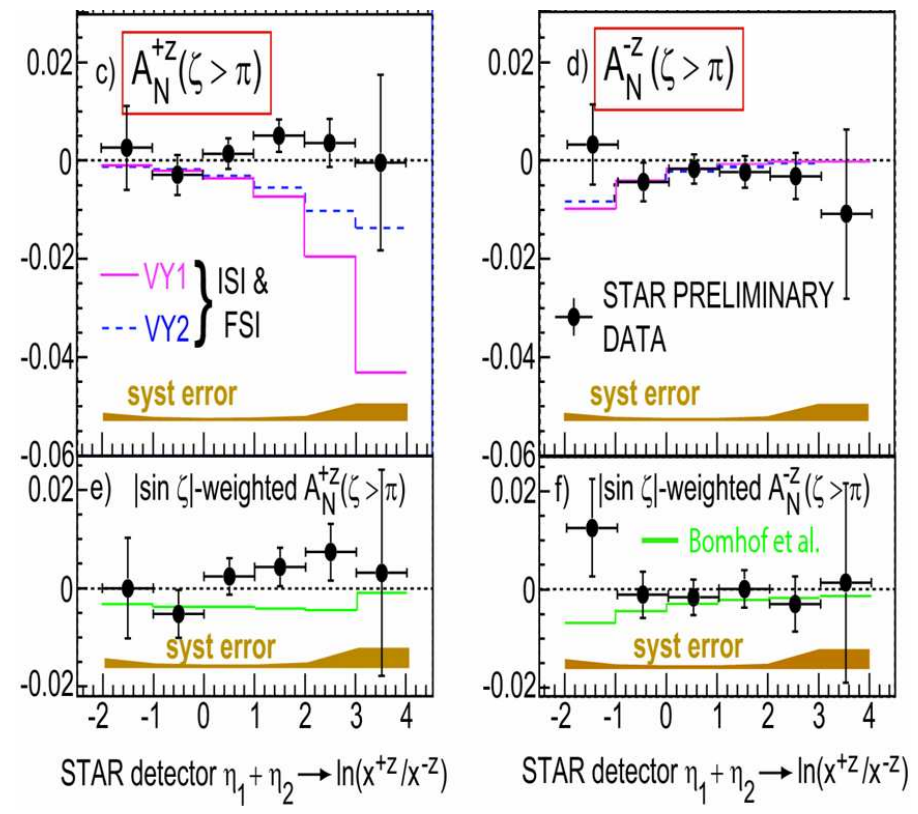

Figure 14: The bisector left-right asymmetry in $p^{\uparrow} p \rightarrow$ jet jet $\mathrm{X}$ measured by STAR [42].

BELLE data. The latest results of this analysis are shown in Fig. 15 [23], with an impressive factor of 19 more statistics compared to the published results [45].

D'Alesio presented a fit of $h_{1}$ and the Collins functions $H_{1}^{\perp}$ from both the $e^{+} e^{-}$ $\cos 2 \phi$ asymmetry (the published data [45]) and the semi-inclusive Collins $\sin \left(\phi_{h}+\right.$ $\phi_{S}$ ) asymmetry (using both HERMES and COMPASS data) [46]. It is interesting to see that all this data can be simultaneously described within the TMD framework. The result supports the above-mentioned observation that the Collins function for favored fragmentation is approximately equal in magnitude to unfavored fragmentation, but of opposite sign. The extracted transversity functions indicate $\left|h_{1}^{d}(x)\right|<\left|h_{1}^{u}(x)\right|$ and opposite sign of $h_{1}^{u}$ w.r.t. $h_{1}^{d}$, see Fig. 16. The question of how to evolve the considered TMD-dependent observables was not yet addressed.

Gamberg presented a model prediction [47] of the $\cos 2 \phi$ asymmetry in unpolar-

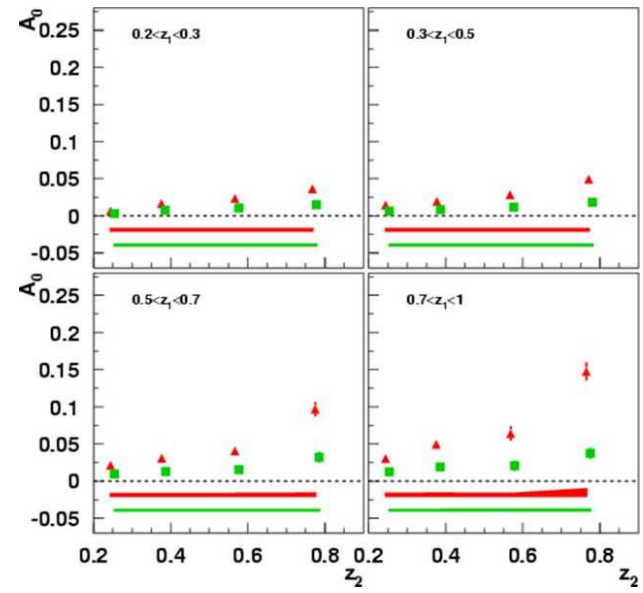

Figure 15: The analyzing power $A_{0}$ of the $\cos 2 \phi$ asymmetry from $547 \mathrm{fb}^{-1}$ of BELLE data [23]. ized semi-inclusive DIS $\left(\propto h_{1}^{\perp} H_{1}^{\perp}\right)$ for the $12 \mathrm{GeV}$ upgrade at JLab, which should provide access to $h_{1}^{\perp}$ (Fig. 17). 

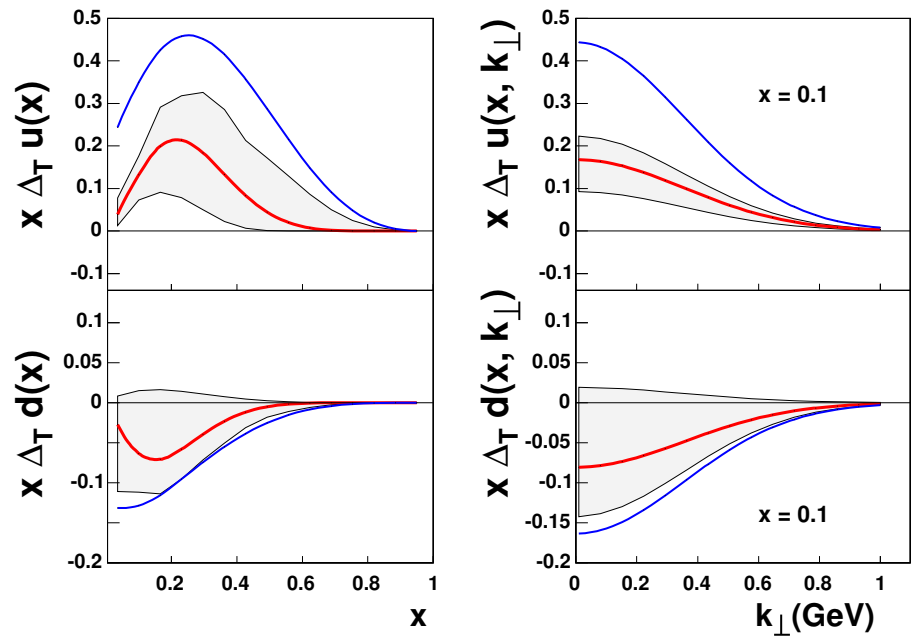

Figure 16: Left panel: the transversity distributions for $u$ and $d$ quarks times $x$, as obtained from a transversity and Collins function fit to BELLE, COMPASS and HERMES data. Right panel: transverse momentum dependence at $x=0.1$. For details cf. [46]

TMDs like $f_{1 T}^{\perp}$ and $h_{1}^{\perp}$ that are odd in $k_{T}$ are spin-orbit coupling quantities, therefore, it is natural to expect a relation with the orbital angular momentum of the quarks, and hence with GPDs. Burkardt [48] pointed out a model-dependent relation between $f_{1 T}^{\perp(1)}$ and the GPD $E$

$f_{1 T}^{\perp(1)}(x) \propto \epsilon_{i j} S_{T}^{i} b_{\perp}^{j} \int d b_{\perp}^{2} \boldsymbol{I}\left(b_{\perp}^{2}\right) \frac{\partial}{\partial b_{\perp}^{2}} E\left(x, b_{\perp}^{2}\right)$

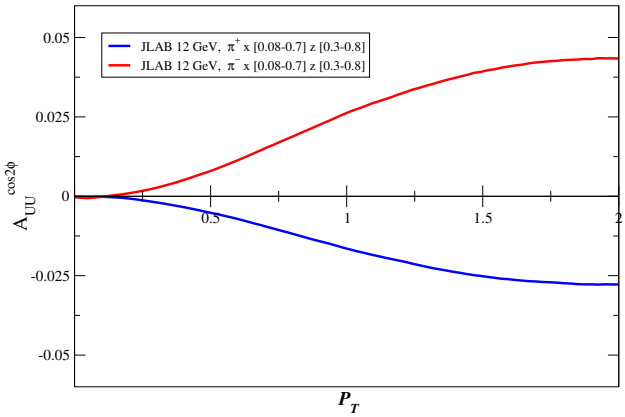

The factor $\boldsymbol{I}\left(b_{\perp}^{2}\right)$ is not analytically calculable, but has to be modeled. Nevertheless, this relation allows to make a qualitative link between the Sivers functions and the

Figure 17: Model prediction of the $\cos 2 \phi$ asymmetry in unpolarized semi-inclusive DIS for JLab@12GeV (updated plot by Gamberg). anomalous magnetic moment of the $u$ and $d$ quarks. Similarly, Burkardt pointed out a relation between $h_{1}^{\perp}$ and a particular combination of two tensor GPDs, for which Hägler presented preliminary lattice results from QCDSF/UKQCD [25], Fig. 18. These are the first lattice results that provide some qualitative information on $h_{1}^{\perp}$ of the pion, indicating that the pion has a surprisingly nontrivial transverse quark spin structure. Metz extended this type of model-dependent, but nontrivial, relations to the other TMDs [49].

\section{Exclusive processes and GPDs}

An outstanding task in solving the 'spin puzzle' of the nucleon is a measurement of the orbital angular momenta of quarks and gluons. For the first time, a possibility to reveal 


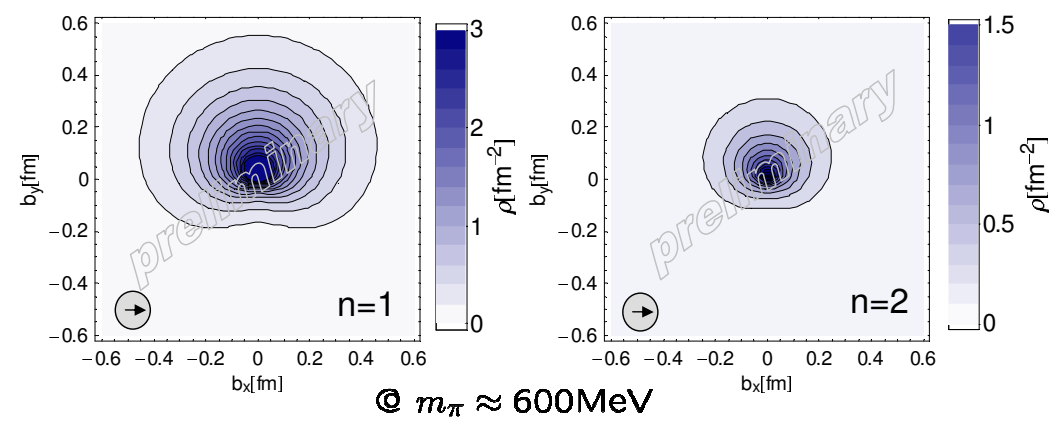

Figure 18: Asymmetric $b_{\perp}$-space distribution of transversely polarized quarks inside a pion from lattice QCD [25].

the total angular momentum carried by the quarks in the nucleon [50] became available within the formalism of Generalized Parton Distributions (GPDs) (see [51] for recent reviews). These functions are related both to the conventional parton densities and to elastic form factors. GPDs provide a wealth of new information as they simultaneously measure longitudinal momentum distribution and transverse location of partons thereby offering a three-dimensional representation of hadrons at the parton level.

GPDs appear in the scattering amplitude of hard exclusive processes. The DVCS process, i.e. the hard exclusive production of a real photon, provides the theoretically cleanest access to GPDs. DVCS amplitudes can be measured most readily through the interference between the Bethe-Heitler process and the DVCS process. A large number of reaction channels can be accessed in hard exclusive meson production. In all cases, polarization observables (e.g. single-spin azimuthal asymmetries) are a powerful tool to obtain information about GPDs.

From the theoretical side, there has been important technical progress in the description of hard exclusive processes, with full NLO results in $\alpha_{s}$ available for most relevant channels, partial NNLO results for Compton scattering and a better understanding of the evolution of GPDs. At DIS2007 Diehl presented such NLO calculations for exclusive meson production at HERA collider and at fixed target kinematics [52]. New avenues have been opened for the parameterization of GPDs: Luiti introduced an alternative to the mathematical ansatz of double distributions in that GPDs are generated from direct constraints from experimental data combined with lattice calculations yielding a model independent extraction [53]. Experimental access to GPDs is very difficult as the count rates for hard exclusive reactions typically drop drastically with increase of the hardness of the process. Nevertheless, there is great progress on the experimental side. HERMES has presented an overview about the so far measured DVCS observables which comprises the full set of azimuthal and single-spin asymmetries w.r.t. the charge and helicity of the lepton beam, and w.r.t. to the spin polarization of the target, either longitudinal or transverse w.r.t. the lepton beam [54]. These results are very promising in view of the greatly improved detection capabilities for exclusive processes with the information from the recoil detector installed early 2006. HERMES also presented the DVCS beam-spin asymmetries measured with a variety of nuclear targets ranging from Deuterium to Xenon [55] which may provide information about the nuclear forces as well as on the modification of nucleon properties in the nuclear medium. Fig. 19, left panel, shows the ratio of the nuclear to free proton DVCS beam-spin asymmetries as a 

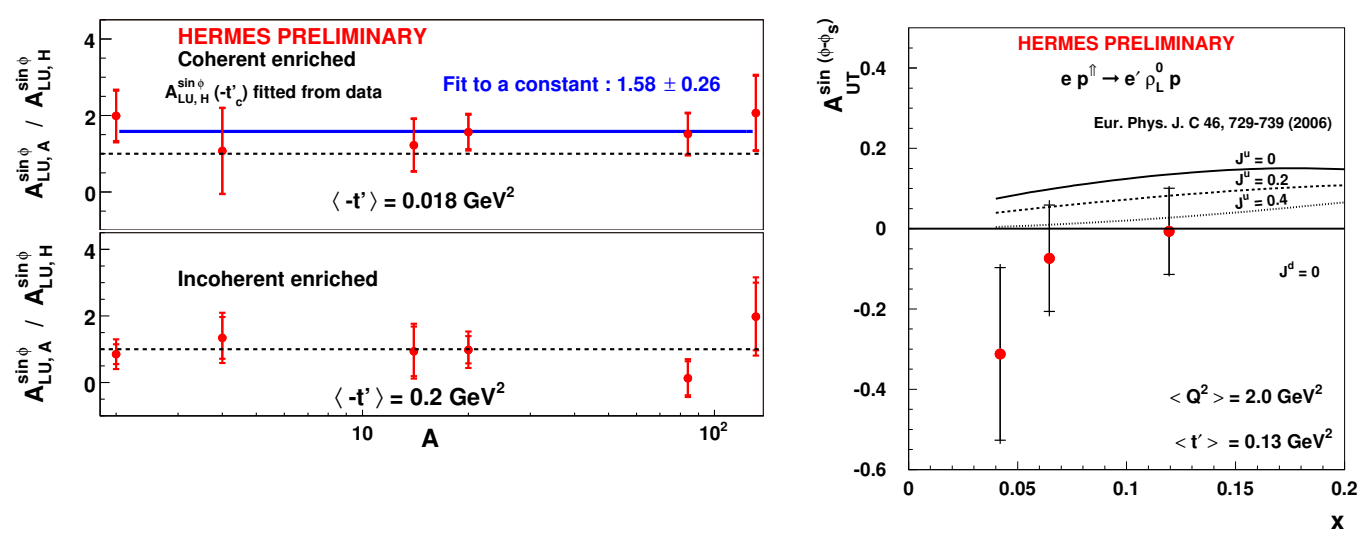

Figure 19: Left panel: The ratio of the nuclear to free proton DVCS beam-spin asymmetries as a function of the atomic mass number $A$ measured by HERMES [55]. Right panel: Transverse target-spin asymmetry for exclusive production of longitudinally-polarized $\rho^{0}$ measured by HERMES [56] and compared to model calculations [57].

function of the atomic mass number $A$. For the coherent region this ratio is predicted [58] to have values ranging from 1.85 to 1.95 for $A=12$ to $A=90$.

The Jefferson Laboratory Hall-A experiment presented a measurement of the DVCS cross-section in the valence quark region on proton and neutron targets [59]. The experiment on the proton provides a strong indication of factorization at $Q^{2}$ as low as $2 \mathrm{GeV}^{2}$, therefore validating a GPD based analysis.

Of particular interest in the context of spin physics is the proton helicity-flip distribution $E^{q}$ which has connection with two crucial aspects of spin physics: transverse polarization effects and the orbital angular momentum $L^{q}$ carried by quarks in the nucleon. Key observables for these studies are transverse target-spin asymmetries in DVCS and in exclusive $\rho^{0}$ production. HERMES has presented preliminary results for both channels $[56,54]$ (see Fig. 19 right panel for the $\rho^{0}$ asymmetry). Their comparison with a model calculation [60] using the total angular momentum of quarks, $J_{q}$, as input parameter in the ansatz for $E^{q}$ shows that these asymmetries are indeed sensitive to $J_{u}$ in the HERMES kinematics. The measurement of the DVCS cross-section on the neutron at Jefferson Laboratory Hall-A experiment [59] provide information about $J_{d}$ using the same GPD model. The complementary constraints on the total angular momenta of up- and down-quarks from both experiments remarkably coincide with recent calculations of $J_{q}$ from lattice QCD [61].

\section{Conclusion and outlook}

Exciting new information has been obtained on the nucleon spin structure from polarized lepton-nucleon and proton-proton scattering. However, a detailed measurement of the gluon polarization remains one of the most important issues in spin physics. Running RHIC at higher energy $(\sqrt{s}=500 \mathrm{GeV})$ will shed more light on this issue.

Transverse spin physics turns out to be a very active and quickly developing field. Important results comprise the first extraction of the transversity as well as of transverse momentum dependent distribution and fragmentation functions like the Sivers distribution 
and the Collins fragmentation function. These achievements can be considered as milestones in the field. They constitute the first step towards a complete description of the partonic structure of hadrons beyond the collinear parton model.

A rich future is expected for the elegant concept of generalized parton distributions (GPDs). Intensive experimental efforts have demonstrated the feasibility of measurements of hard-exclusive reactions in a large variety of channels. It turned out that polarization observables serve as a very powerful tool to access the different GPDs. The interplay between spin degrees of freedom and parton orbital angular momentum will be a key to understand the spin structure of the nucleon.

We thank the organizers for the kind invitation to be part of this successful workshop and furthermore, all speakers of the spin physics sessions for making it such an exciting Working Group.

\section{References}

[1] Slides: http://indico. cern. ch/contributionDisplay.py? contribId=20\&sessionId=2\&conf Id=9499

[2] EMC, J. Ashman et al., Nucl. Phys. B 328, 1 (1989).

[3] HERMES, L. De Nardo, these proceedings; A. Airapetian et al., Phys. Rev. D 75, 012007 (2007).

[4] COMPASS, K. Kurek, these proceedings; V. Y. Alexakhin et al., Phys. Lett. B 647, 8 (2007).

[5] K. Griffioen, these proceedings; CLAS, K. V. Dharmawardane et al., Phys. Lett. B 641, 11 (2006).

[6] K. Slifer, these proceedings.

[7] A. Korzenev these proceedings.

[8] D. de Florian, G. A. Navarro and R. Sassot, Phys. Rev. D 71, 094018 (2005).

[9] J. Blümlein, these proceedings; J. Blümlein, H. Böttcher and A. Guffanti, arXiv:hep-ph/0607200.

[10] D. B. Stamenov, these proceedings;

E. Leader, A. V. Sidorov and D. B. Stamenov, Phys. Rev. D 75, 074027 (2007).

[11] B. I. Ermolaev, these proceedings; B. I. Ermolaev, M. Greco and S. I. Troyan, Eur. Phys. J. C 50, 823 (2007).

[12] P. Liebing, these proceedings.

[13] S. Koblitz, these proceedings.

[14] G. K. Mallot, Proceedings SPIN2006, arXiv:hep-ph/0612055.

[15] K. Okada, these proceedings; PHENIX, A. Adare et al., arXiv:0704.3599 [hep-ex].

[16] B. Jager, A. Schäfer, M. Stratmann and W. Vogelsang, Phys. Rev. D 67, 054005 (2003).

[17] M. Glück, E. Reya, M. Stratmann and W. Vogelsang, Phys. Rev. D 63, 094005 (2001).

[18] R. Fatemi, these proceedings.

[19] F. Simon, these proceedings.

[20] B. Jager, M. Stratmann and W. Vogelsang, Phys. Rev. D 70, 034010 (2004).

[21] P. van der Nat, for the HERMES Collaboration, arXiv:hep-ex/0512019.

[22] C. Schill, these proceedings.

[23] R. Seidl, these proceedings.

[24] M. Radici, these proceedings; F. A. Ceccopieri, M. Radici and A. Bacchetta, arXiv:hep-ph/0703265.

[25] Ph. Hägler, these proceedings.

[26] H. Kawamura, these proceedings; H. Kawamura, J. Kodaira and K. Tanaka, arXiv:hep-ph/0703079.

[27] J. H. Lee, these proceedings.

[28] K. O. Eyser, these proceedings.

[29] S. Heppelmann, these proceedings. 
[30] J. Qiu and G. Sterman, Phys. Rev. Lett. 67, 2264 (1991); Phys. Rev. D 59, 014004 (1999).

[31] Y. Koike, these proceedings; H. Eguchi, Y. Koike and K. Tanaka, Nucl. Phys. B 752, 1 (2006); Nucl. Phys. B 763, 198 (2007).

[32] K. Tanaka, these proceedings; Y. Koike and K. Tanaka, Phys. Lett. B 646, 232 (2007); arXiv:hep$\mathrm{ph} / 0703169$.

[33] D. W. Sivers, Phys. Rev. D 41, 83 (1990); Phys. Rev. D 43, 261 (1991).

[34] A. Bacchetta, these proceedings; A. Bacchetta et al., JHEP 0702, 093 (2007).

[35] D. Boer and P. J. Mulders, Phys. Rev. D 57, 5780 (1998).

[36] A. Airapetian et al. [HERMES Collaboration], Phys. Rev. Lett. 84, 4047 (2000).

[37] M. Diefenthaler, these proceedings.

[38] A. Bressan, these proceedings.

[39] A. Kotzinian, these proceedings.

[40] D. Boer and W. Vogelsang, Phys. Rev. D 69, 094025 (2004).

[41] C. J. Bomhof, these proceedings; C. J. Bomhof, P. J. Mulders, W. Vogelsang and F. Yuan, arXiv:hep$\mathrm{ph} / 0701277$.

[42] J. Balewski, these proceedings.

[43] J. C. Collins, Phys. Lett. B 536, 43 (2002).

[44] D. Boer, R. Jakob and P. J. Mulders, Nucl. Phys. B 504, 345 (1997); Phys. Lett. B 424, 143 (1998).

[45] K. Abe et al. [Belle Collaboration], Phys. Rev. Lett. 96, 232002 (2006).

[46] U. D'Alesio, these proceedings; M. Anselmino et al., Phys. Rev. D 75, 054032 (2007).

[47] L. Gamberg, these proceedings.

[48] M. Burkardt, Nucl. Phys. A 735, 185 (2004).

[49] S. Meissner, A. Metz and K. Goeke, arXiv:hep-ph/0703176.

[50] X. Ji, Phys. Rev. Lett. 78, 610 (1997).

[51] K. Goeke et al., Prog. Part. Nucl. Phys. 47, 401 (2001); M. Diehl, Phys. Rept. 388, 41 (2003); A.V. Belitsky and A.V. Radyushkin, Phys. Rept. 418, 1 (2005).

[52] M. Diehl, these proceedings.

[53] S. Luiti, these proceedings.

[54] A. Mussgiller, these proceedings.

[55] H. Guler, these proceedings.

[56] A. Rostomyan, these proceedings.

[57] F. Ellinghaus et al., EPJC 46, 729 (2006).

[58] V. Guzey and M. Siddikov, J. Phys. G 32, 251 (2006).

[59] E. Voutier, these proceedings.

[60] M. Vanderhaegen, P. A. M. Guichon, M. V. Guidal, Phys. Rev. D 60, 094017 (1999); K. Goeke, M. Polyakov, M. Vanderhaegen, Prog. Part. Nucl. Phys. 47, 401 (2001).

[61] LHPC and MILC collaboration, arXiv:0705.4295 [hep-lat]. 